\title{
Reflets
}

Revue ontaroise d'intervention sociale et communautaire

\section{Appauvrissement des enfants et des familles : défi pour l'Ontario français}

\section{Madeleine Dubois et Brigitte Malenfant}

Volume 3, numéro 1, printemps 1997

Enfance et familles en contexte d'appauvrissement

URI : https://id.erudit.org/iderudit/026148ar

DOI : https://doi.org/10.7202/026148ar

Aller au sommaire du numéro

Éditeur(s)

Reflets : Revue ontaroise d'intervention sociale et communautaire

ISSN

1203-4576 (imprimé)

1712-8498 (numérique)

Découvrir la revue

Citer ce document

Dubois, M. \& Malenfant, B. (1997). Appauvrissement des enfants et des familles : défi pour l'Ontario français. Reflets, 3(1), 9-14.

https://doi.org/10.7202/026148ar

Tous droits réservés (C) Reflets : Revue ontaroise d'intervention sociale et communautaire, 1997
Ce document est protégé par la loi sur le droit d'auteur. L'utilisation des services d'Érudit (y compris la reproduction) est assujettie à sa politique d'utilisation que vous pouvez consulter en ligne.

https://apropos.erudit.org/fr/usagers/politique-dutilisation/ 


\section{Appauvrissement des enfants et des familles: défi pour l'Ontario français}

\section{Madeleine Dubois et Brigitte Malenfant}

"Les prisonniers de toujours doivent être capables de survivre sans cô̂ter trop cher à l'État, les marins du XVIII siècle de tirer sur leurs cordages, les jeunes filles de l'aristocratie pauvre du siècle suivant de lire Racine sans maux de tête et de jouer la Marche turque sans s'évanouir sur leur piano.» (Spitz 1980)

Le choix du mot appauvrissement plutôt que celui de pauvreté comme élément central dans le thème du présent numéro traduit une volonté d'y rassembler des articles, des réflexions et des comptes-rendus illustrant les dimensions multiples et complexes d'une problématique à la fois actuelle et en mouvance. L'appauvrissement touche directement un grand nombre de personnes dans nos communautés et nous interpelle en tant que citoyens et citoyennes. Un rapport du Conseil national du bienêtre social (1997) constate qu'en 1995, cinq millions de personnes, soit $18 \%$ de la population canadienne, vivaient dans la pauvreté. En Ontario, 398000 familles, c'est-à-dire $13 \%$ des familles de la province, vivaient sous le seuil de la pauvreté en 1995, comparativement à 9 \% en 1988.

L'image que l'on se fait de la pauvreté s'articule souvent autour d'une vision statique, se limitant à la dimension économique. Cette vision présente la pauvreté comme un état, dirions-nous même parfois une tare, qui constitue le lot de certaines personnes, particulièrement celles qui sont moins "vaillantes», celles qui sont 
en quelque sorte "nées pour un petit pain", ou qui n'ont pas suffisamment de "coeur au ventre» pour prendre les moyens de se sortir de l'aide sociale. Elle s'inscrit dans le courant de pensée néo-libéral qui suggère que ces individus sont responsables de leurs échecs parce qu'ils n'ont pas réussi à trouver leur intérêt personnel dans une situation de concurrence basée sur la loi de l'offre et de la demande, où l'intervention de l'État en matière sociale doit être minimale.

Loin de prétendre attaquer sur plusieurs fronts à la fois tous les mythes liés à la pauvreté, nous proposons ici quelques angles pouvant servir de cadre à une lecture de la problématique de l'appauvrissement, et plus particulièrement à l'ancrage de celle-ci au sein de divers contextes ou de pratiques ayant une certaine résonance pour les réalités et les défis multiples de la population franco-ontarienne.

Dans le contexte actuel de compressions budgétaires, de restructuration et de rationalisation, souvent motivées par la quête de profits corporatifs outranciers, le phénomène de l'appauvrissement résulte fréquemment d'une insuffisance de revenu causée par la perte d'emploi, le chômage de longue durée, le manque d'expérience, particulièrement chez les jeunes, la pénurie d'emplois stables et le foisonnement d'emplois précaires ou à temps partiel, en majorité mal rémunérés. Les modifications dans les programmes sociaux aux niveaux fédéral et ontarien laissent un filet de sécurité sociale dangereusement troué, contribuant à fragiliser davantage la précarité des circonstances de vie d'un grand nombre de personnes. Une des participantes à un projet d'entraide communautaire a bien évoqué cette situation au cours de l'entrevue avec Reflets, présentée ici: «Je n'ai pas toujours été dans cette situation. Avant je vivais assez bien. Mais en raison de circonstances de vie, $\mathrm{j}$ 'ai dégringolé». En effet, personne n'est à l'abri de "circonstances de vie». Nous savons toutefois que le fait de vivre une séparation ou un divorce, ou encore de quitter un conjoint violent, augmente la vulnérabilité des femmes face à l'appauvrissement et à ses multiples implications. Comme en témoigne l'article de Carole Giguère, les défis auxquels sont confrontées les femmes tentant d'échapper à la violence conjugale 
se complexifient davantage suite aux compressions du gouvernement Harris dans les budgets et les services qui leur sont destinés. Les jeunes subissent aussi les conséquences de la fragilisation d'un tissu social marqué par la pénurie d'emplois. Dans un article traitant du programme Avenir, conçu pour faciliter le cheminement des jeunes dans leurs démarches vers l'emploi, Maxim Gravel fait état d'un taux de chômage plus élevé chez les jeunes que dans l'ensemble de la population ontarienne. Dans ce programme, on note de plus un pourcentage plus élevé de participation de jeunes francophones que de jeunes anglophones, compte tenu du pourcentage de la population francophone en Ontario. Par ailleurs, le décrochage scolaire et l'analphabétisme, qui demeurent des préoccupations importantes pour les Ontariennes et les Ontariens dont la langue maternelle est le français, contribuent aussi à la vulnérabilité des jeunes et des familles. Colette Lacroix et Yasmine Nedjar décrivent deux programmes conçus par la Magie des lettres à l'intention des jeunes enfants et des familles, cherchant à agir sur certains facteurs menant aux décrochage scolaire, plus particulièrement au sein de familles où l'un ou l'autre des membres est analphabète.

La situation géographique peut aussi constituer un facteur de vulnérabilité face aux aléas de l'emploi et à l'appauvrissement pour une partie de la population franco-ontarienne. Dans le Nord plus particulièrement, les francophones habitent souvent dans des villes à industrie unique, s'exposant ainsi aux bouleversements personnels et sociaux causés par une fermeture d'usine, une grève, le déménagement d'une partie importante des effectifs ou des activités d'une compagnie. Marge Reitsma-Street, Adje van de Sande et François Boudreau dressent un portrait de la pauvreté dans la région de Sudbury, où l'industrie minière constitue le moteur de l'économie. L'analyse des statistiques de cette région, où plus du tiers de la population a le français comme langue maternelle, révèle un taux de pauvreté plus élevé que celui de la moyenne ontarienne, alors que le taux de pauvreté infantile est plus élevé chez les francophones que chez les anglophones de la région. L'article présente quelques-uns des moyens entrepris pour sensibiliser la population sudburoise à cette réalité, afin de la 
situer au coeur des enjeux sociaux faisant appel à la solidarité de l'ensemble de la communauté.

Le recours à des qualificatifs tels que démunis, marginalisés et nécessiteux attribués à des personnes dites pauvres porte à occulter la créativité et le dynamisme des systèmes de débrouillardise des personnes vivant avec une insuffisance de revenu et à minimiser l'importance de leurs contributions sociales. Ce ne sont pourtant pas les exemples qui manquent pour illustrer leur participation et leurs nombreuses contributions à la vitalité des réseaux sociaux. Dans un article analysant les principes fréquemment évoqués pour justifier la mise en oeuvre de cuisines collectives, Manon Mongrain et Cécile Coderre avancent que l'implication des femmes dans celles-ci dépasse largement le cadre de la sécurité alimentaire, et donc de la pauvreté économique, et contribue à l'éclosion et à la valorisation de potentialités ainsi qu'au renforcement du tissu social. Cet article trouve aussi écho dans les propos rapportés par les femmes dans l'entrevue avec Reflets. Certaines d'entre elles parlent en effet de leur participation à des cuisines collectives comme d'un tremplin vers une plus grande autonomie. En plus d'offrir des exemples concrets des pratiques créatives et du dynamisme de nombreux groupes d'entraide, l'article de Michèle Kérisit et de Nérée St-Amand illustre l'importance pour ces groupes d'être enracinés au sein des communautés auxquelles ils se rattachent. Ils soulignent de plus la nécessité pour les organismes communautaires de créer des espaces de convivialité pour que les familles pauvres s'engagent dans un processus d'actualisation de leurs forces et de prise en charge de leurs ressources. Philippe Lelièvre décrit un programme destiné aux enfants de la région de Prescott-Russell impliquant une collaboration étroite entre les ressources communautaires, les parents des enfants ainsi que les médias en vue d'élaborer des activités de prévention, de dépistage et de soutien pour les familles et les jeunes.

Un autre thème abordé dans le contexte plus large de l'appauvrissement touche à l'importance de repenser nos pratiques. Que ce soit au sein d'organismes communautaires ou de type plus institutionnel, nos interventions peuvent s'articuler autour de valeurs sociales dominantes qui alimentent et reproduisent certains 
préjugés systémiques contribuant à l'assujetissement et à l'exclusion plutôt qu'à la conscientisation et à l'inclusion. Confrontées à des logiques d'efficacité et à des rationalisations restreignant de plus en plus les marges de manoeuvre et les espaces de résistance possibles, nos pratiques glissent, parfois à notre insu, vers des approches qui ont tendance à minimiser l'importance accordée à la parole des usagers et des usagères, à prendre en charge des problèmes ou des personnes plutôt qu'à favoriser l'émergence de potentiels. Dans cette perspective, Marie Drolet analyse la signification attribuée à l'empowerment dans le contexte de diverses approches. Dans le travail auprès des familles, elle fait valoir la nécessité d'une vision plus globale, qui contextualise dans une perspective sociale les problèmes auxquels elles sont confrontées, par rapport à une approche axée sur la gestion de problèmes individuels, où l'empowerment participerait à une rhétorique de surresponsabilisation. Dans un texte présentant quelques réflexions se dégageant des premiers jalons d'une recherche portant sur le milieu associatif en Ontario français, Rachid Bagaoui met en perspective le point de vue d'intervenantes et d'intervenants confrontés aux répercussions du désengagement de l'État. Il discute de stratégies amorcées comprenant la mise en place de nouveaux partenariats qui orientent les objectifs des milieux associatifs vers des pratiques de solidarités renouvelées entre les diverses composantes de la francophonie ontarienne. De leur côté, Brigitte Malenfant et Louise Delisle du Conseil de planification sociale d'Ottawa-Carleton décrivent les résultats d'une enquête auprès de la clientèle des services communautaires dans la région ayant pour but de connaître les besoins à l'égard des services. Elles font part de diverses suggestions face aux moyens visant à faciliter l'accès aux services socio-communautaires ainsi que des stratégies d'action pour faire face aux coupures des services en français.

En acceptant d'entreprendre la planification de ce numéro, nous avons dû confronter nos craintes face aux chances de réussite d'un projet d'écriture sur la question de l'appauvrissement des enfants et des familles en Ontario français. Conscientes de la pénurie d'écrits sur le sujet, nous espérions encourager la rédaction d'articles visant à faire connaître les projets au sein de nos diverses 
communautés. Il s'agissait aussi d'amorcer une réflexion sur quelques-uns des nombreux enjeux liés à l'appauvrissement, dans un contexte social où la proportion de personnes et de familles pauvres s'accroît d'année en année, augmentant sérieusement durant chaque crise économique sans toutefois diminuer pendant les périodes de reprise. Les textes recueillis pointent vers des pistes d'analyse riches de réflexions et illustrent des pratiques créatives axées sur la solidarité et sur les potentialités des personnes engagées dans des actions communautaires en Ontario français.

\section{Bibliographie}

CONSEIL NATIONAL DU BIEN-ÊTRE SOCIAL (1997). Profil de la paureté, Ottawa.

SPITZ, P. (1980) «Violence silencieuse, famine et inégalités». Revue internationale de sciences sociales, vol. XXX, no 4, 919-941 